\title{
What affects the performance of Merger and Acquisition in China: A
}

\section{review for Chinese literature}

\author{
Xiao-feng Shang ${ }^{1, \text { a }}$ \\ ${ }^{1}$ Business School, Shandong Normal University, Jinan 250014, China. \\ axiaofeng_sh1108@163.com
}

Keywords: M \& A, Enterprise Performance, Empirical Reference, China.

\begin{abstract}
Since 20 century, Merger and Acquisition has become an instantaneous hot spot in China, given that it is one of the most important activities of asset allocation. Therefore, many scholars in China have conducted a large amount of surveys from different views. My study summarizes the extant related Chinese literature from the following five aspects, such as the characteristics of the acquirer, target company characteristics, M \& A mode, afterwards management and the external environment. This study provides a clear framework to present the influential factors on the performance of $\mathrm{M} \& \mathrm{~A}$. In addition, it also points out the extant defects of researches and gives some advice to the academic field, which can provide the guidance to the further study.
\end{abstract}

\section{Introduction}

Merger and Acquisition activities mainly refer to a corporation action of purchasing the shares or assets of another company. Although enterprises can grow and expand though internal capital accumulation, the way of external mergers and acquisitions is undoubtedly more direct and fast. In 21 century, companies are more likely to choose Mergers and Acquisitions for diverse growth and expansion, which has become a common and a typical method in China. Since Belt and Road Forum for International Cooperation was held successfully, China has experienced a rapid economic growth. In terms of M \& A market, in China, it has been much more booming than ever before, as for a leading way of optimizing allocation of resources in the capital market, and overall pass rate reaches to 93.06 percent. According to the data from the Shanghai stock exchange, it can conclude that the number of public corporations, which had accomplished M \& A activities in Shanghai, added up to 864 times in 2017, increasing 45 percent compared to last year, and total transaction value amounted to 920 billions RMB. The industrial M \& A and innovation projects gradually occupy the mainstream position while the shell listing continues to cool down with the cross-border M \& A increasing significantly. In addition, there are 74 corporations completing the acquisition of significant assets, involving in deal value about 250 billions RMB. With the increasing M \& A events, the main business operations of investment bank in China have extended from IPO businesses to mergers businesses.

Prior to these years, due to Acquisition of Interest Protection System aiming to conserve the interests of minority shareholders, numerous shareholders were crazy about purchasing the stocks, issued by the listed companies, which led to stock price rising and presented a trend that the enterprise could flourish after merger activities. Therefore, a few of corporations tended to emulate without thinking deliberately and $\mathrm{M} \& \mathrm{~A}$ became an instantaneous hot spot. However, influenced by the ability of management and coordination, the accuracy of accounting information provided by 
target company and the integration after takeover, the effect of $\mathrm{M} \& \mathrm{~A}$ event is uneven: after $\mathrm{M} \& \mathrm{~A}$ events, some corporations have acquired the desired effect as predict that they successfully integrate the resource and attain maximum profit, the other are absolutely opposite and even assume enormous loss because of lacking independent innovation ability. Based on these above, M \& A market gradually emerges chaotic situation and administrators are at a loss what to do. After that, there have been a large number of scholars gradually emerging to concentrate on the factors which have influence on the $M \& A$ performance, and then they share the results to the public. Most of them use quantitative method, data processing, empirical analysis, to list just a few, in their dissertations, so as to prove which shows positive effect or negative effect.

Given that thousands of researches could be utilized as the reference, corporations are able to correctly adjust their $\mathrm{M} \& \mathrm{~A}$ scheme in order to attain the idealized profit. Based on the estimation of M \& A value and the trend of stock value in 2007, it can be predicted that the difference of M \& A estimation will be parochial in the prime market and the secondary market, that short-term arbitrage space will be compassed, and that the attitude towards $\mathrm{M} \& \mathrm{~A}$ activities will return to rationality so that the stock value reflection will return to value investment. Obviously, guided by the policy of industrial mergers and acquisitions and the influence of the double-wheel driving pattern of interest constrain, the pace of audition begins to accelerate and audit efficiency is improved. The merger and reorganization Committee is mainly focused on continued profitability of the standard company, legal compliance, trading impartiality, profit forecast and so on.

My study attempts to reorganization and review extant papers on mergers and acquisition. In order to better differentiate between which are positive factors and which are negative factors, these factors are roughly divided into five aspects, characteristics of the acquire, target company characteristics, Mergers and Acquisitions mode, post-merger factors and external environment, which can present the influential factors in a clear framework. This essay will provide the reference value for the supervision department to manage, the government to optimize the market environment and the enterprises to merge.

\section{Development}

\subsection{The characteristics of the acquirer}

There have been a great number of scholars studying the influence on $M \& A$ activities from the perspective of the acquirer. First of all, from the point of control power, some scholars have found out that the bigger control power of the merger company, the more the discourse power of making the merger decision. In one aspect, some prefer to carry out empirical investigations to search issues on the basis of the political relevance, where the government has a large leading power on operating process. They bear out that the stronger political relevance, the worse performance, which results from the different aims between the corporation and the government and a large amount cost is spent on maintaining this relationship. [1,2] However, for some cultural industries, this correlation shows the reverse effect. This is because the property right of this special industry is extremely different from other businesses, which assumes the responsibility of shaping values and moral norms. Thus, since it began to develop, the governments have provided financing and political supports and this political relevance helps the cultural industry enhance the performance. [3] In another aspect of control power, other scholars also realize that corporation's ownership structure is worth delving and they usually divide this structure into two areas, the nature of shareholders and the concentration of shareholders. In the field of the former, the fact is ubiquitous, that different aims between the companies and the individuals. If the personal benefits are closely related to the company's interest, managers prefer to make decisions based on the perspective of sustainable 
development, which efficiently enhances the performance. [4] When it comes to the concentration of shareholders, Ou Yang \& Yuan [5] also draw a conclusion that it is negatively related to the performance. Now that $\mathrm{M} \& \mathrm{~A}$ activities are usually regarded as speculative actions in the companies with a high concentration of equity. In addition to the control influence, others pay attention to company's capital liquidity----amount of free cash flow held. It is undeniable that M \& A requires a sufficient financial support and companies with a large amount funds have more active selection rights. However, when holding ultra-cash flow, managers prefer to invest low-return projects rather than distributing them to shareholders, resulting in excessive investments. Consequently, such excessive free cash flow turns out to damage the M \& A performance. [6])

Belonging to above classification, agent issue is a focus of the scholar research, which is one of the decisive factors of the $\mathrm{M} \& \mathrm{~A}$ performance. In one aspect, the extant problem, some scholars find out, is that if directors and executives merge into one position, it fails to achieve ideal performance. [7] They find it is because this fact leads to enhance CEO rights and reduce the efficiency of board supervision. In addition, Yang [8] reports that the performance is positively correlated to the ratio of independent directors and further explains that independent directors have no interest in the company and they can take full account of the interest of all shareholders.

\subsection{Target company characteristics}

As merger and acquisition activities involve two parties, some scholars also have doubted whether the nature of the target companies can affect the performance of $M \& A$, based on different stages. Before mergers and acquisitions, the acquirer needs to determine whether the target company is worth the merger and whether they can achieve the expected consequence. Therefore, financial indicators, such as ratio of assets to liabilities, net sales rare, growth rate of business and the like, are commonly regarded as the effective test methods, which can directly reflect the financial and operating conditions of target enterprises. [9] Then, if the target company is judged to be worthy of purchasing, the acquirer should also consider whether the accounting information provided by the target company is true, which is determined by the quality of accounting information. In that the higher quality contributes to restrict the blind, arrogant, speculative investment, the quality becomes extremely important. Pan \& Yv [10], combing short-term market performance(CAR) and long-term accounting performance (ROE, EBIT, ROA), point that the higher quality of accounting information contributes to the better performance. Even if the above two points are taken into account and have a positive impact, there are many other factors coordinated as well after the merger and the one of most widely studied factors is culture discrepancy. Because if the buyers think much highly of their own values, which leads to neglect the cultural distinction of target companies and make the counterpart have a strong resistance. Therefore, when they are integrating, cultural intensity is negatively correlated with acquisition performance and even causes cultural conflict. [11]

\section{$2.3 \mathrm{M} \&$ A mode}

In addition to analyze the main bodies of $M \& A$, the course of $M \& A$ activity has many decisive factors, affecting performance. If all of the above influential factors are considered and proven to be beneficial to the $M \& A$, the performance will still have different effects based on choosing means of $\mathrm{M} \& \mathrm{~A}$, for its own resources and capabilities will change rapidly as the corporation develops to a specific stage. For instance, He [12] finds that if the enterprises are in the growth and maturity period, they should give priority to asset acquisition, compared with the equity transfer, given that they have gained the real operating right, which allows them to integrate and utilize resources. Moreover, there is another existing merger way, such as horizontal, vertical and mixed M \& A. Fan \& Yuan [13] find that the horizontal M \& A should be carried out in the growing companies, now 
that by this way, the growing companies can take up a larger market share and attain much profits. After deciding apposite M \& A strategy, the acquirer needs to pay the corresponding price for buying out the target companies. However, the seemingly simple buying and selling activity will have different results, due to the enterprise needs to contrive the payment method. Compared with cash payment, stock payment method turns out to ameliorate corporate governance and optimize performance, since it enhances the balance between the leading shareholders and other ones. [14]

\subsection{Afterwards management}

After the $M \&$ A activities, it is pervasive that lacking the integration section leads to the failure and the underlying reason for the fact is that the acquirer lacks the ability to integration, such as management integration, enterprise culture integration and strategic integration. In one aspect, after $\mathrm{M} \& \mathrm{~A}$, the acquirer needs to regulate a systematic principle to form cohesion and complementary advantages, since differences between two companies exist in staff quality, departmental function and management system. For, example, Xie \& Tian [15], based on the management integration, stress that the stronger the management ability, the better the acquisition performance. Moreover, the possibility is absolutely little, that both two enterprises have the same corporation culture. At this point, some scholars [16]find that cultural integration seems to be vital to the buyer. If these two various cultures are not able to coalesce, it can obscure the faculties to understand enterprise spirit and to obey the norm, which can eliminate the performance. Most importantly, they also report that strategic integration is the most crucial factors to increase the performance, on the ground that before $\mathrm{M} \& \mathrm{~A}$, these companies have distinctive market environment, development history and marketing method, without an effective coordination, it is impossible that all of resource are coherently operated as a whole, and even the all of the above integration are impossible to be successfully advanced.

\subsection{The external environment}

During these years, the phenomena is emergent that a large number of cross-border merger and acquisition activities are beginning to occur in China, which means that companies have more chance to occupy the market share. In the meanwhile, the corresponding external environment, having a profound impact on M \& A activities, evokes much attention. When focusing on the mergers happening in domestic, in terms of the taxation, some scholars [17,18] discover that there is a significant positive correlation between present tax competition differences and enterprise crossregion performance. They find it is because whichever the company the acquirer chooses, such differences offer more institutional arbitrage and cross-regional coordination to the buyer, promoting the optimization of industrial structure. In addition, in term of local innovation development, Chen \& $\mathrm{Wu}$ [19] prove that the difference of regional innovation level is in proportion to the performance, in that the bigger the difference between these two enterprises, the greater potential for integration and promotion to both companies, which leads to leave more room for facilitating the operating performance. Furthermore, when focusing on the cross-border merger and acquisition cases, most scholars [20,21] have conducted empirical investigations on several factors damaging the performance, such as formal system and informal system. They find it is because two companies are located in various countries whose have different legal system, economic principle and cultural background, which gives rise to increase the management difficulty after $\mathrm{M} \& \mathrm{~A}$.

\section{Conclusion}

By consulting and collecting the literature, the researches on the $\mathrm{M} \& \mathrm{~A}$ performance have been extremely inclusive, which most of literature are from the characteristics of the acquirer, target 
company characteristics, M \& A mode, afterwards management and the external environment. However, there are still some deficiencies about research direction, research method and empirical analysis existing in extant study.

First of all, afterwards management should be further studied. The success of M \& A depends largely on afterwards management. Many cases tell us it is dangerous if acquirers only buy the target companies but can't make them in and produce synergy effects. For example, in 2018, 50\% of Motorola' staffs will be laid off by Lenovo, as the share of market of Lenovo continues to decrease. This is because internal teams have interest conflicts and business integration has short board. Therefore, $M \& A$ integration is worth being investigated, especially in strategy integrate and management integrate. In addition, some scholars pay more attention to the domestic activities, whereas the field, cross-border mergers and acquisitions, has not been sufficiently studied. Based on some particular factors, such as the culture, policy and work style, the companies frequently face extraordinary challenges, which they have ever met before. Without fully analyzing, the acquirer usually turns to feel panic and fails to solve the difficulty, which leads to damage the performance.

Secondly, the data used in existing study come from common database, which means these data has been utilized numerous times and it is hard to generate a new finding. Recently, questionnaire has been more and more popular among scholars. However, when conducting a questionnaire, it is easy for objects to bring subjective feelings into answer the question, which leads to a biased result. If the scholars can use other database or change their research angle, it will enhance the efficiency of data. In addition, the sample size researchers investigate needs to be large enough so that the result concluded from the questionnaire becomes more objective and reliable.

Finally, endogeneity is still a problem for empirical study to watch out. More instrument variables and DID method need to be used. Furthermore, most of scholars would like to measure the performance using parallel indicators, such as ROA, ROE, and some parameters about profits. However, profits may not be the only target for $\mathrm{M} \& \mathrm{~A}$. Therefore, the method needs to be more flexible, such as using comprehensive scoring methods. If scholars are able to think of some original standards, their literature will bring more reference value to the field.

\section{Reference}

[1] Wang Yvchun, Liang Hongji, Qin Yun. Influence of political relevance on the performance of SME merger and acquisition based on the nature of property right and the perspective of institutional environment, 2014.

[2] Zhao Xi, Tian Yuan. Merger performance and Enterprise-enterprise correlation - based on empirical study on listed companies in China, 2016.

[3] Pan Ailing, Wang Linlin. Property rights attribute, political relevance and merger and acquisition performance of cultural enterprise, 2015.

[4] Liu Junhao. Investment institutions ' shareholding ratio, accounting conservatism and merger performance, 2017.

[5] Ouyang Luwei, Yuan Xianfeng, Wen Mei, Zhang Xueying. The empirical study on the effect of ownership structure on listed companies ' merger and acquisition performance, 2011.

[6] Zhang Fangfang, Liu Shulian. Cash holding, merger and acquisition decision and the performance, 2015. 
[7] Pan Ying, Wang Kai. The empirical study of the relationship between board governance and merger performance of listed companies, 2014.

[8] Yang Yuan. The empirical study of the relationship between agent issue and the performance on listed companies, 2012.

[9] Hu Linhao, Zhao Yiwen. Enterprise growth, strategic coordination and the performance of the acquirer, 2017.

[10] Pan Hongbo, Yv Honggui. The quality of the accounting information of the target, property rights attribute and the performance of the merger and acquisition, 2014.

[11] Wang Yan, Kan Shou. The culture of enterprise and the merger and acquisition performance, 2014.

[12] He Dengjiao. Enterprise life cycle, merger and acquisition type and the performance, 2016.

[13] Fan Conglai, Yuan Jing. The empirical study of the relationship between the Growth industry, mature industry, declining industry and the merger and acquisition performance, 2002.

[14] Zhou Shaoni, Wang Huitong. The payment method, enterprise governance and the merger and acquisition performance, 2015.

[15] Xie Jigang, Tian Fei, Ren Qiao, Han Shanshan. The ability to merger and acquisition governance - A new perspective to explain the failure of merger and acquisition, 2009.

[16] Liu Ruizhi, Xu Chaoyang, Zhou Chang. The empirical study of the relationship between the integration and the merger and acquisition performance, 2014.

[17] Yv Mingtao, Pan Ailing, Li Bin. The difference of tax competition level, the ability to tax governance and the cross-regional performance, 2017.

[18] Wang Yi, Zhang Jinxin, Yv Jing. Whether merger and acquisition can bring about the optimization of capital structure - empirical evidence from Chinese listed companies, 2015.

[19] Chen Zhigang, Wu Jisen, Gui Li. The empirical study of the relationship between the innovation level and the merger and acquisition performance, 2016.

[20] Zhang Chi, Yv Pengyi. The difference of institutional distance to the performance of transnational mergers and acquisitions in China - based on the comparison of horizontal and vertical merger and acquisition, 2017.

[21] Li Qiang. Research on the effect of institutional distance on the performance of Chinese enterprise transnational merger and acquisition, 2015. 